\title{
MicroRNA-155 induces protection against cerebral ischemia/reperfusion injury through regulation of the Notch pathway in vivo
}

\author{
TIANPENG JIANG, SHI ZHOU, XING LI, JIE SONG, TIANZHI AN, \\ XUEQIN HUANG, XIUQIN PING and LIZHOU WANG \\ Department of Radiology, Affiliated Hospital of Guizhou Medical University, Guiyang, Guizhou 550004, P.R. China
}

Received September 18, 2017; Accepted March 1, 2018

DOI: $10.3892 /$ etm.2019.7590

\begin{abstract}
RNA (miR)-155 has been demonstrated to participate in the regulation of endothelium during cerebral ischemia. In the present study, it was aimed to investigate the molecular mechanism of miR-155 in the regulation of cerebral ischemia/reperfusion (I/R) injury with middle cerebral artery occlusion (MCAO) in mice. The MCAO model was established in C57BL/6 mice. Transfection of miR-155 mimics and miR-155 inhibitors was performed to alter the expression of miR-155. The level of miR-155 was measured by RT-qPCR analysis. The western blotting results demonstrated that deletion of miR-155 increased the expression of Notch1, intracellular Notch receptor domain (NICD) and hairy and enhancer of split-1 (Hes1) levels. In addition, the percentage of terminal deoxynucleotidyl-transferase-mediated dUTP nick end labeling-positive cells and caspase- 3 levels were decreased following treatment with a miR-155 inhibitor compared with the Pre-IR group. Notably, disrupting miR-155 also increased nitric oxide (NO) production and the expression of endothelial NO synthase (eNOS), leading to downregulation of brain water content and Evans blue levels. However, overexpression of miR-155 restored all these changes to similar levels observed in the cerebral I/R injury group. The expressions of Notch1, NICD and Hes1 were also decreased to the cerebral I/R injury condition. In conclusion, a novel mechanism was identified for abrogating normal NO production and eNOS expression via the aberrant expression of the Notch signaling pathway, a mechanism that may be modulated by miR-155. Together, these results reveal important functions of miR-155 in regulating the Notch signaling pathway of the nervous system, and a potential role for miR-155 as a crucial therapy target for cerebral stroke.
\end{abstract}

Correspondence to: Professor Lizhou Wang, Department of Radiology, Affiliated Hospital of Guizhou Medical University, 28 Guiyi Road, Guiyang, Guizhou 550004, P.R. China

E-mail: wanglizhougz@163.com

Key words: cerebral ischemia/reperfusion injury, microRNA-155, Notch pathway, endothelial nitric oxide synthase, nitric oxide

\section{Introduction}

Ischemic stroke represents one of the major causes of neuronal injury and disability, which may have increased morbidity and high mortality rates during the next 20 years, especially in developing countries (1). The overall cumulative risk of mortality is $47 \% 5$ years after an ischemic stroke (2). Reperfusion following ischemic stroke may induce secondary brain damage, leading to neurological dysfunction and cerebral endothelial injury, thereby increasing cerebrovascular permeability and blood-brain barrier (BBB) leakage (3). This is known as cerebral ischemia/reperfusion ( $\mathrm{I} / \mathrm{R})$ injury, which provides a notable challenge for general surgeries and the treatment of ischemic stroke (4). As such, there is an urgent requirement to elucidate the development of the molecular mechanisms underlying cerebral I/R injury and to identify novel therapeutic strategies to improve clinical outcomes.

The Notch pathway is a highly conserved signaling system that controls cellular self-renewal and survival during the development of various tissues (5). To date, 4 Notch receptors (Notch1-4) and 5 Notch ligands (Jagged1/2 and Delta-like 1/3/4) have been identified to serve important roles in the Notch signaling pathway (6). A series of proteolytic cleavages initiated by the binding of Notch ligands to receptors are coupled with the release and translocation of the intracellular Notch receptor domain (NICD) to the nucleus, which activates the transcription of Notch-targeted genes and regulates cell fate decisions (7). In the central nervous system (CNS), Notch is associated with the differentiation, maturation, and functional maturation of neural progenitor cells (8). The association of Notch with I/R conditions in the cerebrum has previously been demonstrated, and increased Notch1 expression has been observed during cerebral ischemia (9-11). Furthermore, Notch has been reported to be implicated in the regulation of endothelial cell proliferation and vessel growth $(12,13)$. However, the underlying mechanism of Notch in cerebral I/R injury associated with BBB dysfunction remains to be elucidated.

microRNAs (miRNAs or miRs) are a class of endogenous, non-coding RNA molecules comprising 19-23 nucleotides, which can modulate gene expression by binding with the 3'-untranslated region (UTR) of target mRNA molecules $(14,15)$. miRNAs serve important roles in physiological 
and pathologies processes, which are associated with ischemic stroke (16-18). As a key inflammation-related miRNA, miR-155 expression is significantly altered by cerebral ischemia $(19,20)$. miR-155 has also been demonstrated to increase vascular endothelial permeability $(21,22)$. Therefore, understanding the role of miR-155 and its mechanisms in cerebral I/R injury may provide an informative approach to treating this disease.

The aim of the present study was to investigate the role and molecular mechanism of miR-155 in the regulation of cerebral I/R injury with middle cerebral artery occlusion (MCAO) in mice, by assessing the function of BBB and the apoptosis index, and measuring the expression of miR-155, Notch1, Jagged1 and hairy and enhancer of split-1 (Hes1) in the ischemic cortex. These results demonstrated a close association between Notch signaling and miR-155 in cerebral I/R injury, indicating a potential target for the treatment of ischemic stroke.

\section{Materials and methods}

Animals. A total of 120 male C57BL/6 mice were purchased from the Experimental Animal Center of Guizhou Medical University (Guiyang, China). Specific pathogen-free mice were housed under steady state microenvironment conditions (temperature, $20 \pm 2^{\circ} \mathrm{C}$; 12-h light/dark cycle; $95 \%$ humidity), with ad libitum access to food and water. All procedures were approved by the Animal Care and Research Committee of The Affiliated Hospital of Guizhou Medical University (Guiyang, China).

Experimental protocol. To evaluate the expression change of miR-155 during I/R injury, 16 8-month-old C57BL/6 male mice (weight, 20-25 g) were randomly divided into the following two groups ( $\mathrm{n}=8$ in each): Sham-operated group (sham) and I/R group (Pre-IR). The Pre-IR group was observed continuously for $\leq 24 \mathrm{~h}$ following $\mathrm{I} / \mathrm{R}$.

A conditional miR-155 knockout approach was performed to reveal the role of the Notch signaling pathway in ischemic brain injury. A miR-155 inhibitor (miR-155 ${ }^{-/}$) and miR-155 mimics (miR-155 $5^{+/+}$) were used. A total of 120 8-month-old C57BL/6 mice (weight, 20-25 g) were randomly divided into the following six groups $(n=20$ in each): Sham, Pre-IR, sham+miR-155 inhibitor (miR-155/-sham), Pre-IR+miR-155 inhibitor (miR-155 ${ }^{-/}$Pre-IR), sham+miR-155 mimics (miR-155 $5^{+/+}$sham), Pre-IR+miR-155 mimics (miR-155 $5^{+/}$Pre-IR). Mice in Sham groups were subjected to surgical procedures without arterial occlusion, whereas mice in the Pre-IR groups were subjected to MCAO.

Lentiviral transfection in mice. To modify the expression of miR-155 in the mouse model, purified lentiviral particles containing miR- $155^{+/+}$or $\mathrm{miR}-155^{-/-}$were obtained from Shanghai GenePharma Co., Ltd. (Shanghai, China). The

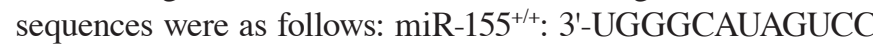
UAAUCGUAAUU-5'; miR-155 ${ }^{-/}:$3'-UGCAUAUAAUGCUAA AGCAUUAA-5'; control miRNA: 3'-UAAACAUGUACGCAU GCAUAGCU-5'. Prior to administration, mice were anesthetized and fixed on a stereotactic frame, lentivirus constructs (miR-155 ${ }^{+/+}, \mathrm{miR}^{-155^{-/}}$and scrambled control; $\left.10^{9} \mathrm{TU} / \mathrm{ml}\right)$ were mixed with the cationic lipid Polybrene ( $4 \mu \mathrm{g} / \mu \mathrm{l}$; Sigma-Aldrich; Merck KGaA, Darmstadt, Germany) and incubated at $37^{\circ} \mathrm{C}$ for $15 \mathrm{~min}$. Subsequently, each mouse was slowly administered $7 \mu 1$ mixture over 20 min via right intracerebroventricular injection. At 14 days following viral vector injection, MCAO procedure was performed on these mice.

$M C A O$. The MCAO model was established in C57BL/6 mice according to the methodology used in a previous study (23). Briefly, mice were anesthetized with $4 \%$ chloral hydrate (Sigma-Aldrich; Merck KGaA), and the left common, internal (ICA) and external carotid arteries (ECA) were carefully isolated. A 6-0 nylon suture was inserted into the ECA stump, gently injected into the ICA and stopped at the opening of the middle cerebral artery (MCA). The distance from the bifurcation of ICA/ECA to MCA was $10 \mathrm{~mm}$. When the injection had been in place for $90 \mathrm{~min}$, nylon sutures were gently removed from the ICA and reperfusion was performed (22). Body temperature was maintained at $37^{\circ} \mathrm{C}$ during the surgical procedure. Sham-operated mice received the same surgical procedure without insertion of the nylon suture.

Evaluation of neurological scores. Following cerebral I/R injury, mice were assessed for neurological deficits and scored by three blinded examiners as described previously (24). Points were awarded in the grading system as follows: 0 , no deficit; 1 , forelimb weakness; 2 , circling to affected side; 3 , partial paralysis on affected side; and 4, no spontaneous motor activity. MCAO mice were allowed to recover for $24 \mathrm{~h}$ prior to evaluation. Neurological scores were evaluated at 24, 48 and $72 \mathrm{~h}$ following MCAO.

Staining with 2-3-5-triphenyl terazolium chloride (TTC). At $24 \mathrm{~h}$ following MCAO, five mice in each group were anesthetized with intraperitoneal chloral hydrate $(1 \mathrm{~g} / \mathrm{ml}$ in acetonitrile; $0.04 \mathrm{ml} / 100 \mathrm{~g}$ body weight; Sigma-Aldrich; Merck $\mathrm{KGaA}$ ) and sacrificed via decapitation. Mouse brains were harvested. The sections were fixed in $10 \%$ formaldehyde for a minimum of $24 \mathrm{~h}$ at $4^{\circ} \mathrm{C}$ in the dark. Sections were then coronally sliced into $2-\mathrm{mm}$ slices. The slices were stained with $2 \%$ TCC (Sigma-Aldrich; Merck $\mathrm{KGaA}$ ) at $37^{\circ} \mathrm{C}$ for $20 \mathrm{~min}$ in the dark. The infarct brain area was represented by white coloring, whereas normal brain tissues were stained red. The infarct area was measured using MetaMorph software (Molecular Devices, LLC, Sunnyvale, CA, USA). The infarct volume of each slice was calculated as follows: $V=t \mathrm{x}(A 1$ $+A 2+A 3+A 4+A 5)-(A 1+A 2) \times t / 2$ (A represents the infarct area of the slice, $\mathrm{t}$ represents the slice thickness). The lesion volume is presented as a volume percentage of the lesion compared with the contralateral hemisphere as previously described (25).

Histological analysis. Brains from five mice in each group were fixed in $4 \%$ paraformaldehyde overnight at $4{ }^{\circ} \mathrm{C}$, and subsequently washed with $0.9 \%$ saline and dehydrated. Following embedding the brain in paraffin wax, $5-\mu \mathrm{m}$ sections were cut and stained with hematoxylin and eosin (HE) for $8 \mathrm{~min}$ at $37^{\circ} \mathrm{C}$. Slides were viewed and imaged under an Olympus light microscope (Olympus Corporation, Tokyo, Japan) at a magnification of $\mathrm{x} 400$.

Evans blue dye extravasation method. Evans blue (2\% in saline; $0.4 \mathrm{ml} / 100 \mathrm{~g}$ body weight; Sigma-Aldrich; 
Merck KGaA) was injected into the jugular vein of mice and allowed to circulate for $24 \mathrm{~h}$. Subsequently, mice were sacrificed and brains were quickly removed and weighed, then homogenized in $2.5 \mathrm{ml} \mathrm{PBS}$ and centrifuged at $10,000 \mathrm{x} \mathrm{g}$ for $30 \mathrm{~min}$ at $4^{\circ} \mathrm{C}$. Evans Blue was measured using a spectrophotometer (Thermo Fisher Scientific, Inc., Waltham, MA, USA) at $610 \mathrm{~nm}$.

Brain water content. Following sacrifice, five mouse brains in each group were quickly removed and weighed (wet weight). The brains were then dried at $120^{\circ} \mathrm{C}$ for $24 \mathrm{~h}$ and weighed again (dry weight). The percentage of water content was calculated as follows: (wet weight-dry weight)/wet weight x100.

Nitric oxide $(\mathrm{NO})$ release. NO production was measured using an NO assay kit (cat. no. S0021; Beyotime Institute of Biotechnology, Haimen, China) according to the manufacturer's protocol. The assay was based on the Greiss test (26). Mice were euthanized, brains $(n=5)$ were quickly removed, and tissues were homogenized in radioimmunoprecipitation assay lysis buffer (Beyotime Institute of Biotechnology) and centrifuged at $15,000 \mathrm{x}$ g for $15 \mathrm{~min}$ at $4^{\circ} \mathrm{C}$. Following centrifugation, $50 \mu \mathrm{l}$ supernatant or standard $\mathrm{NaNO}_{2}$ were mixed with $50 \mu \mathrm{l}$ Griess reagent I and $50 \mu \mathrm{l}$ Griess reagent II in a 96-well plate for $10 \mathrm{~min}$ at $4^{\circ} \mathrm{C}$. Nitrite was measured using a spectrophotometer (Thermo Fisher Scientific, Inc.) at $560 \mathrm{~nm}$ and quantified by the colorimetric MTT assay (Sigma-Aldrich; Merck KGaA).

Terminal deoxynucleotidyl-transferase-mediated dUTP nick end labeling (TUNEL) staining. Apoptotic cell death was detected using a TUNEL staining kit (Roche Applied Science, Penzberg, Germany) according to the manufacturer's instructions. Paraffin-embedded brain sections were deparaffinized in dimethylbenzene, rehydrated with gradient alcohol and digested with Proteinase K $(20 \mu \mathrm{g} / \mathrm{ml}$; Sigma-Aldrich; Merck KGaA) for $30 \mathrm{~min}$ at $37^{\circ} \mathrm{C}$. Following pretreatment, sections were incubated in a solution containing Biotinylated Nucleotide mix and Terminal Deoxynucleotidyl Transferase at $37^{\circ} \mathrm{C}$ for $1 \mathrm{~h}$. Antifade fluorescence mounting media was added to the coverslip and the edges were sealed with nail polish. Samples were observed and images were captured under fluorescence microscopy. Five fields of view were observed at a magnification of $x 400$.

Reverse transcription-quantitative polymerase chain reaction $(R T-q P C R)$. Total RNA was extracted from mouse brains $(n=5)$ using TRIzol ${ }^{\circledR}$ reagent (Invitrogen; Thermo Fisher Scientific, Inc.) according to the manufacturer's protocol. Reverse transcription was performed using universal primers and the PrimeScript ${ }^{\mathrm{TM}}$ RT reagent kit (Takara Biotechnology Co., Ltd., Dalian, China) at $37^{\circ} \mathrm{C}$ for $15 \mathrm{~min}$ and $85^{\circ} \mathrm{C}$ for $5 \mathrm{sec}$. Transcripts were quantified by qPCR using a BioRad CFX96 real-time PCR detection system with SYBR-Green $\mathrm{iQ}^{\mathrm{TM}}$ Supermix (both from Bio-Rad Laboratories, Inc., Hercules, CA, USA). The PCR reaction was performed at $95^{\circ} \mathrm{C}$ for $3 \mathrm{~min}$, followed by 40 cycles of $95^{\circ} \mathrm{C}$ for $12 \mathrm{sec}$ and $60^{\circ} \mathrm{C}$ for $40 \mathrm{sec}$. An internal control (GAPDH) was used to normalize the expression of the target genes. The following primers were used to amplify the gene fragments, and were available from Genebank (https://www.ncbi.nlm.nih.gov/genbank/): miR-155, forward, 5'-GCTTCGGTTAATGCTAATCGTG-3' and reverse, 5'-CAGAGCAGGGTCCGAGGTA-3'; Notch1, forward, 5'-TCAATGTTCGAGGACCAGATG-3' and reverse, 5'-TCACTGTTGCCTGTCTCAAG-3'; Hes1, forward, 5'-AGC CAACTGAAAACACCTGATT-3' and reverse, 5'-GGACTT TATGATTAGCAGTGG-3'; and GAPDH, forward, 5'-CAT GGCCTTCCGTGTTCCTA-3' and reverse, 5'-CCTGCTTCA CCACCTTCTTGA-3' (Shanghai GenePharma Co., Ltd., Shanghai, China). The relative quantification of the gene expression was calculated using the comparative quantification cycle $(\mathrm{Cq})$ method (27). Mean $\mathrm{Cq}$ values and deviations between the duplicates were calculated; $\triangle \mathrm{Cq}=(\mathrm{Cq}$ mRNA-Cq mRNA control $)$, and fold change $=\left(2^{-\Delta \mathrm{Cq}} \mathrm{mRNA} / 2^{-\Delta \mathrm{Cq}} \mathrm{mRNA}\right.$ control). RT-qPCR analysis of the mRNA levels of miR-155 was measured in mouse brains at 6,12 and $24 \mathrm{~h}$ following MCAO.

Western blotting. Mouse brains $(\mathrm{n}=5)$ were homogenized in RIPA lysis buffer (Beyotime Institute of Biotechnology) by the BCA method, and homogenates were centrifuged at $12,000 \mathrm{x} \mathrm{g}$ for $15 \mathrm{~min}$ at $4^{\circ} \mathrm{C}$. Supernatants were collected and stored at $-80^{\circ} \mathrm{C}$. Protein samples $(50 \mu \mathrm{g} /$ lane) were separated by SDS-PAGE on $10 \%$ gels and proteins were transferred onto polyvinylidene difluoride membranes. Following the transfer, the membranes were blocked for $2 \mathrm{~h}$ with $5 \%$ nonfat dry milk at room temperature. Membranes were incubated overnight at $4^{\circ} \mathrm{C}$, with the following primary antibodies: Anti-endothelial NO synthase (eNOS; cat. no. 32027; 1:1,000), anti-caspase3 (cat. no. 9662; 1:1,000), anti-Notch1 (cat. no. 3608; 1:1,000), anti-Hes1 (cat. no. 11988; 1:1,000), anti- $\beta$-Actin (cat. no. 3700; 1:2,000; all Cell Signaling Technology, Inc., Danvers, MA, USA) and anti-NICD (cat. no. ab8925; 1:1,000; Abcam, Cambridge, UK). Following incubation with the primary antibodies, membranes were washed with Tris-buffered saline containing $0.05 \%$ Tween-20 (TBST) 3 times, and subsequently incubated with appropriate horseradish peroxidase-conjugated anti-rabbit secondary antibodies $\lg \mathrm{G}$ (cat. no. 14708; 1:2,000; Cell Signaling Technology, Inc.) at room temperature for $2 \mathrm{~h}$. Membranes were visualized using an enhanced chemiluminescence assay kit (Merck KGaA). Protein bands were digitally scanned and then analyzed using ImageJ software (version 1.48; National Institutes of Health, Bethesda, MD, USA).

Statistical analysis. Data are presented as the mean \pm standard deviation of at least three experiments. Statistical analyses were performed using SPSS 22.0 (IBM Corp., Armonk, NY, USA). Differences among groups were analyzed using by one-way analysis of variance with post hoc Student-Newman-Keuls test. $\mathrm{P}<0.05$ was considered to indicate a statistically significant difference.

\section{Results}

Effects of cerebral I/R injury in mice. To determine the effects of cerebral I/R injury on mice, neurological scores and TTC staining were performed. As presented in Fig. $1 \mathrm{~A}$ and $\mathrm{B}$, there was no notable neurological deficit and infarct exhibited by the sham group. Compared with the sham group, the MCAO 


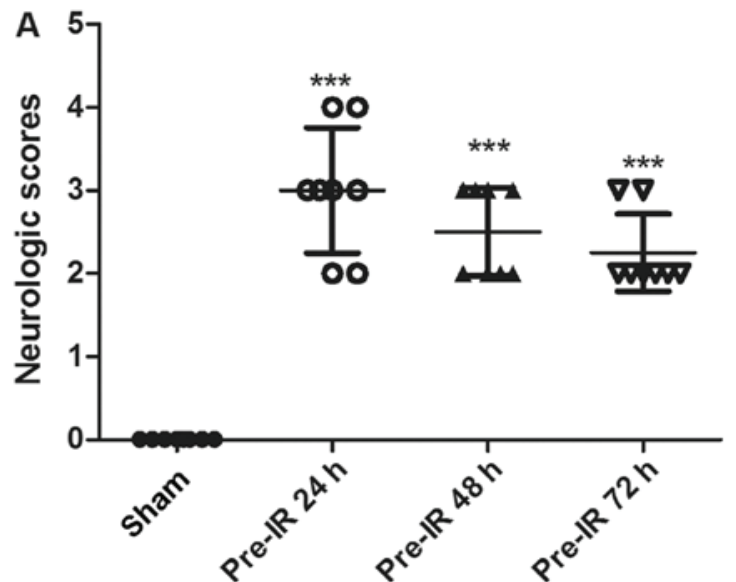

B
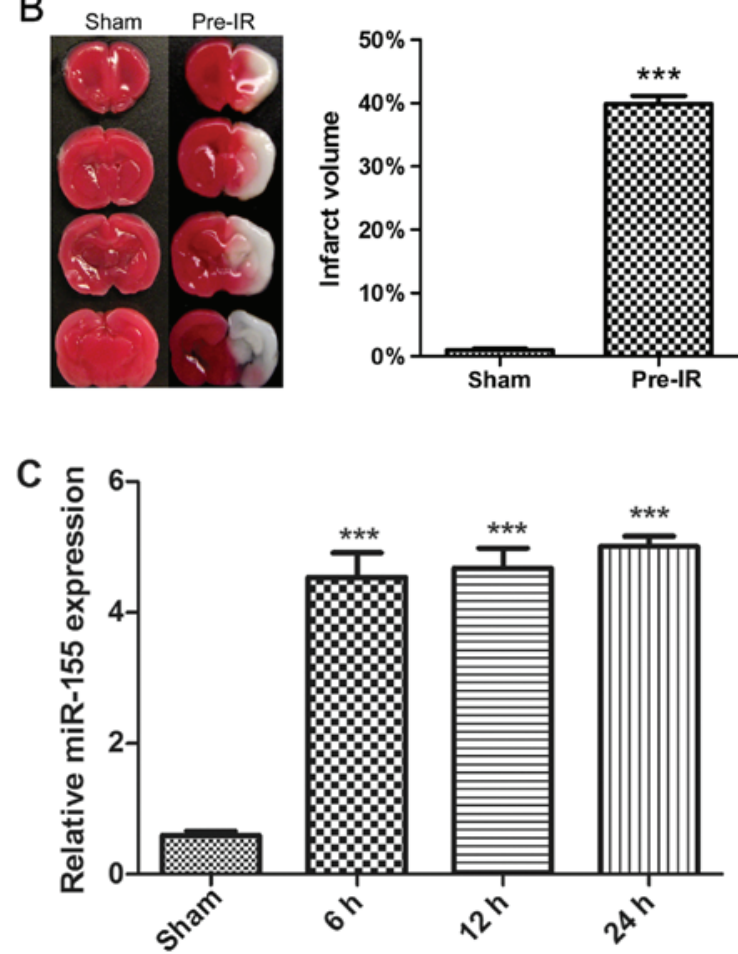

Figure 1. Effects of cerebral I/R injury in mice. (A) Neurological scores were evaluated at 24, 48 and $72 \mathrm{~h}$ following MCAO $(\mathrm{n}=8)$. (B) Staining with 2-3-5-triphenyl terazolium chloride indicated brain infarct at 6, 12 and $24 \mathrm{~h}$ following MCAO ( $n=3)$. (C) Reverse transcription-quantitative polymerase chain reaction analysis of miR-155 mRNA levels in mouse brains at 6,12 and $24 \mathrm{~h}$ following MCAO $(\mathrm{n}=5)$. Data are expressed as the mean \pm standard deviation. ${ }^{* * *} \mathrm{P}<0.001$ vs. sham. I/R, ischemia reperfusion; MCAO, middle cerebral arterial occlusion; $\mathrm{miR}$, microRNA.

mice in the Pre-IR group exhibited a significant increase in neurological deficit and infarct $(\mathrm{P}<0.001)$. Furthermore, the neurological deficit was alleviated over time.

To evaluate cerebral I/R injury on the regulation of miR-155 expression, miR-155 mRNAs were analyzed via RT-qPCR. As presented in Fig. 1C, cerebral I/R injury significantly increased the synthesized levels of miR-155 mRNAs in brains, in comparison with the sham group $(\mathrm{P}<0.001)$. The maximal expression of miR-155 was observed at $24 \mathrm{~h}$.

Effects of miR-155 on BBB following cerebral I/R injury in mice. To determine the effects of miR-155 on the brain histopathology, HE staining was evaluated. As presented in Fig. 2A, the brain cortical tissue was intact in the sham group. In accordance with this, the neurons were integral and cellular structures were clear. However, the cortical tissues exhibited congestion and edema, and the number of nerve cells were markedly decreased in the Pre-IR group. In the miR-155/-Pre-IR group, neurons were arranged in neat rows, and hyperemia and edema vanished. Intriguingly, the histopathologic changes observed in the miR-155/++Pre-IR group appeared similar to those in the Pre-IR group, which indicated that the cortical tissues in this group exhibited serious hyperemia and edema accompanied by decreased cortical neurons.

To investigate the effects of miR-155 on BBB permeability, Evans blue staining and brain water content were evaluated. As presented in Fig. 2B and C, the content of Evans blue and brain water exhibited a significant increase in the Pre-IR group compared with the sham group $(\mathrm{P}<0.001)$. However, miR-155/- significantly decreased the Evans blue and brain water levels in the miR-155 $5^{-/}$Pre-IR group compared with the Pre-I/R group. Compared with the miR- $155^{-/}$Pre-IR group, the content of Evans blue and brain water in the miR-155 $5^{+/}$Pre-IR group were significantly ameliorated $(\mathrm{P}<0.01)$.

To evaluate the effects of miR-155 on vascular endothelial functions, NO content and the protein expression eNOS were assayed. As presented in Fig. 2D and E, the content of $\mathrm{NO}$ and the expression of eNOS in brains were significantly decreased in the Pre-IR group, compared with the sham group $(\mathrm{P}<0.001)$. However, miR-155 ${ }^{-/}$significantly ameliorated NO and eNOS levels $(\mathrm{P}<0.001)$. Compared with the $\mathrm{miR}-155^{-/}$Pre-IR group, the content of NO and the expression of eNOS in miR-155 $5^{+/+}$Pre-IR group were significantly decreased to levels similar to those of the Pre-IR group $(\mathrm{P}<0.001)$.

Effects of miR-155 on apoptosis following cerebral I/R injury in mice. As presented in Fig. 3A, apoptotic cells (green) were almost absent in ischemic area of the sham group. The percentage of apoptotic cells was significantly increased in ischemic areas following MCAO procedure compared with the sham group $(\mathrm{P}<0.001)$. The percentage of TUNEL positive cells was significantly decreased in the miR-155 $5^{-/}$Pre-IR group compared with the Pre-I/R group $(\mathrm{P}<0.001)$. Compared with the miR-155/-Pre-IR group, the TUNEL positive cells in the miR-155 ${ }^{+/}$Pre-IR group were significantly ameliorated to levels similar to those of the Pre-IR group $(\mathrm{P}<0.001)$.

As presented in Fig. 3B, the expression of caspase- 3 in mouse brains was significantly increased in the Pre-IR group compared with the sham group $(\mathrm{P}<0.001)$. However, miR-155 significantly decreased the caspase-3 levels in miR-155 ${ }^{-/}$Pre-IR group compared with the Pre-I/R group $(\mathrm{P}<0.01)$. In comparison with the miR-155 ${ }^{-/}$Pre-IR group, the caspase-3 level in the miR-155 ${ }^{+/}$Pre-IR group was significantly ameliorated to a level similar to that of the Pre-IR group $(\mathrm{P}<0.01)$.

Effects of miR-155 on the Notch signaling pathway following cerebral I/R injury in mice. To investigate whether miR-155 serves a role in the Notch signaling pathway, the expression of Notch1, NICD, Jagged1 and Hes1 were analyzed via western blotting. As presented in Fig. 4A and B, the protein levels of Notch1 were increased in increased in the Pre-IR group in comparison with the sham group $(\mathrm{P}<0.001)$. However, compared with the Pre-IR group, the levels of Notch1 in the 

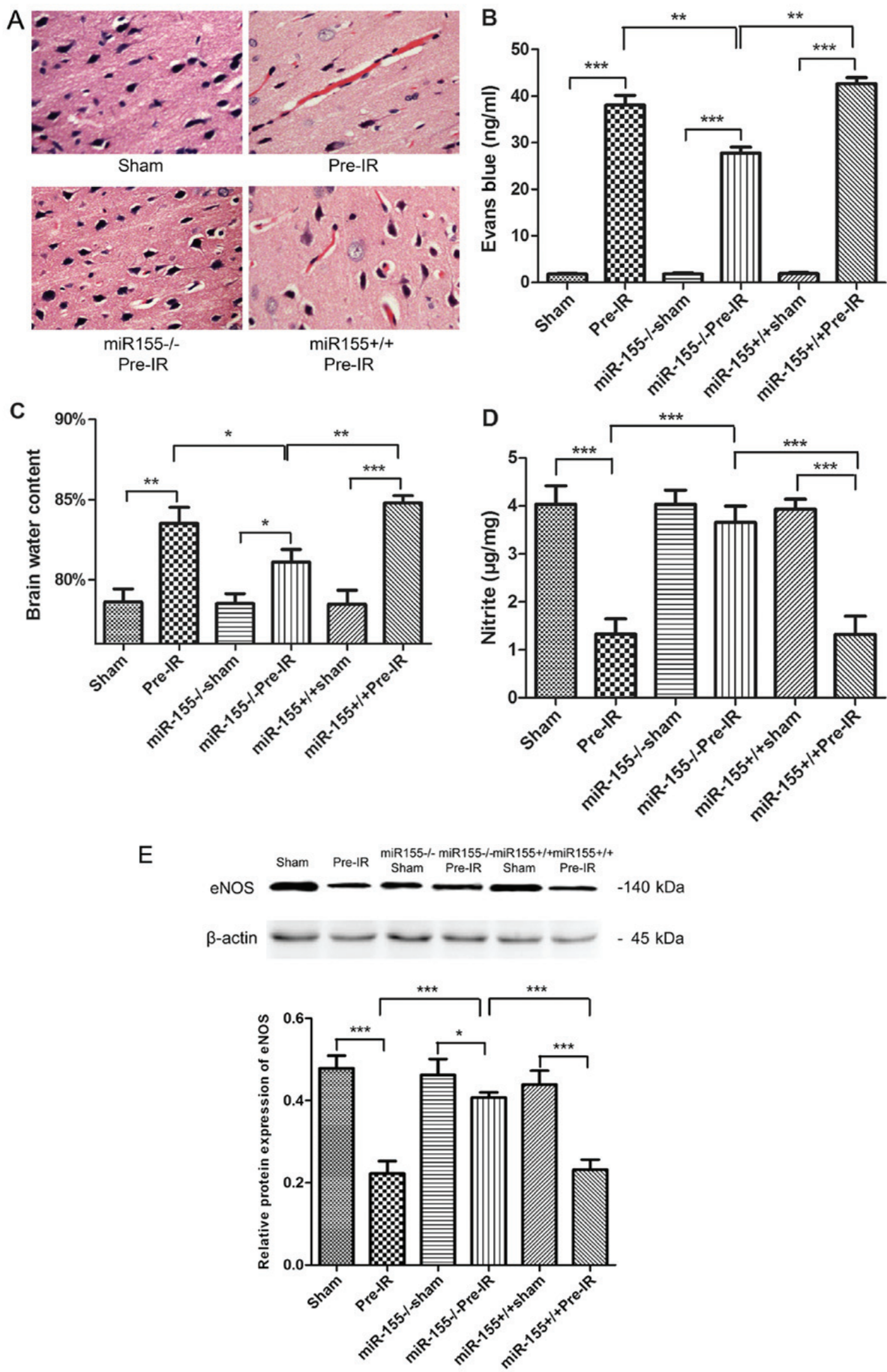

Figure 2. Effects of miR-155 on brain endothelial BBB following cerebral I/R injury in mice. (A) Hematoxylin and eosin staining (magnification, $\mathrm{x} 40$ ), (B) brain water content and (C) Evans blue staining were quantified as indications of BBB integrity at $24 \mathrm{~h}$ following MCAO (n=5). (D) NO levels in brains were tested at $24 \mathrm{~h}$ following MCAO $(\mathrm{n}=5)$. (E) Western blot analysis of the eNOS protein levels in brains at $24 \mathrm{~h}$ following MCAO ( $=5$ ). Data are expressed as the mean \pm standard deviation. ${ }^{*} \mathrm{P}<0.05,{ }^{* *} \mathrm{P}<0.01,{ }^{* * *} \mathrm{P}<0.001 . \mathrm{miR}$, microRNA; BBB, blood-brain barrier; I/R, ischemia reperfusion; MCAO, middle cerebral arterial occlusion; NO, nitric oxide; eNOS, endothelial NO synthase; miR-155 ${ }^{-/}$, miR-155 inhibitor; miR-155 $5^{+/}$, miR-155 mimics. 

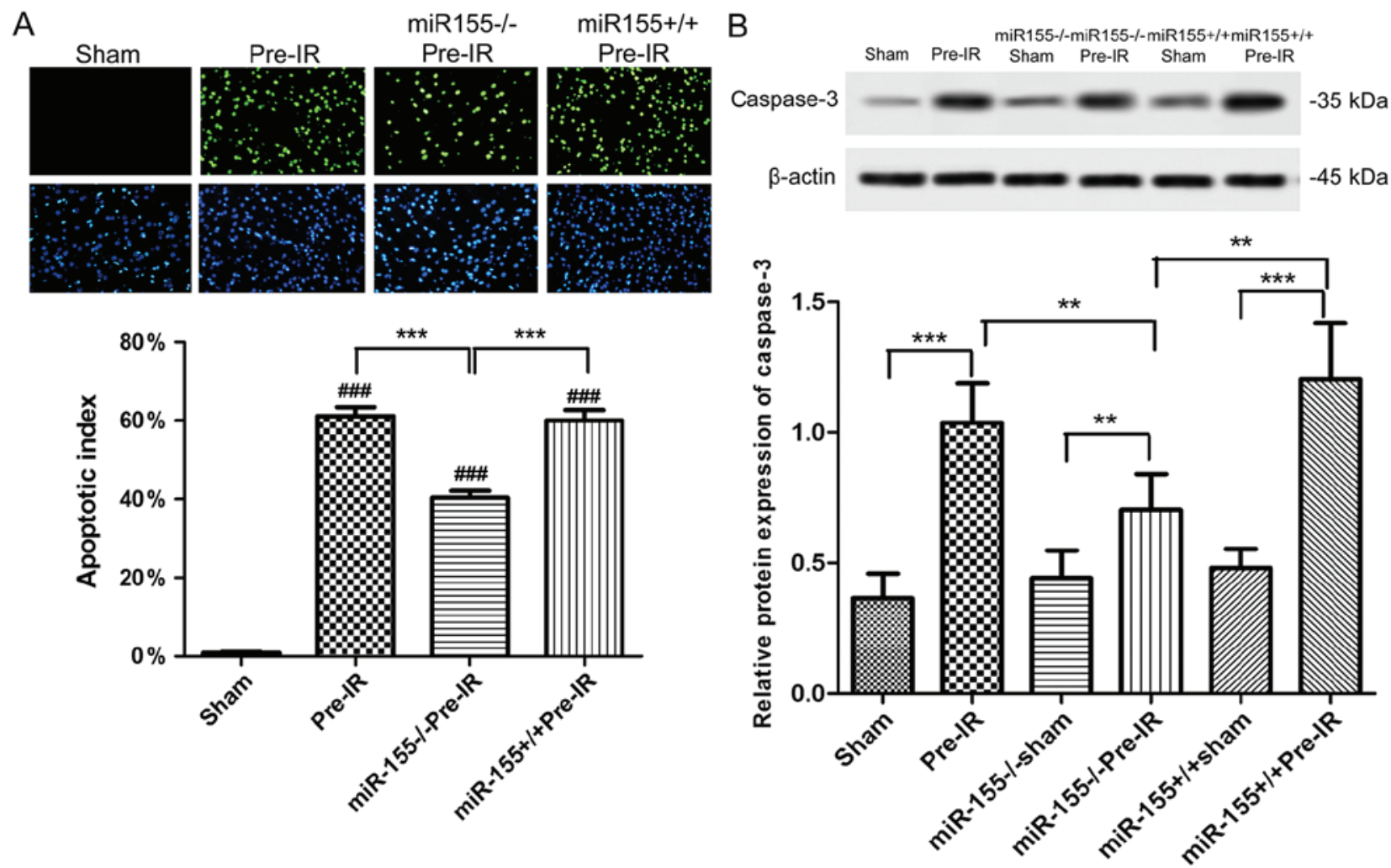

Figure 3. Effects of miR-155 on apoptosis following cerebral I/R injury in mice. (A) TUNEL staining (magnification, x200) demonstrated the neuronal apoptosis following cerebral I/R injury ( $\mathrm{n}=5$ ). Apoptotic cell death was detected using a TUNEL staining kit. The green staining indicated TUNEL-positive cells and the blue staining indicated cell nucleus. (B) Western blot analysis of the caspase-3 protein levels in mouse brains at $24 \mathrm{~h}$ following middle cerebral arterial occlusion $(\mathrm{n}=5)$. Data are expressed as the mean \pm standard deviation. ${ }^{* *} \mathrm{P}<0.01,{ }^{* * *} \mathrm{P}<0.001 ;{ }^{* \# \#} \mathrm{P}<0.001$ vs. sham. miR, microRNA; I/R, ischemia reperfusion; miR-155 ${ }^{-/}$, miR-155 inhibitor; miR-155 ${ }^{+/+}$, miR-155 mimics; TUNEL, terminal deoxynucleotidyl-transferase-mediated dUTP nick end labeling.

miR-155 ${ }^{-/}$Pre-IR group were significantly increased $(\mathrm{P}<0.05)$. In comparison with the miR-155 ${ }^{-/} \mathrm{Pre}-\mathrm{IR}$ group, the expression of Notch1 in the miR-155 ${ }^{+/}$Pre-IR group was significantly decreased to similar levels to the Pre-IR group $(\mathrm{P}<0.05)$. As presented in Fig. 4A, C and D, changes in NICD and Hes1 levels were similar to those of Notchl.

To investigate whether miR-155 has a role in the Notch signaling pathway, the Notch1 and Hes1 mRNA levels were analyzed via RT-qPCR. As presented in Fig. 4E, the mRNA levels of Notch1 was significantly increased in the Pre-I/R group in comparison with the sham group $(\mathrm{P}<0.001)$. However, compared with the Pre-IR group, the mRNAs level of Notch1 in the miR-155 ${ }^{-/}$Pre-IR group was significantly increased $(\mathrm{P}<0.001)$. In comparison with the miR-155 ${ }^{-1} \mathrm{Pre}-\mathrm{IR}$ group, the levels of Notch1 in miR-155 ${ }^{+/}$Pre-IR group was signifcantly decreased $(\mathrm{P}<0.001)$. As presented in Fig. 4F, the trend of Hes1 changes was similar to that of Notch1.

\section{Discussion}

The CNS is a part of the nervous system that contains a large number of microvascular cells, which permits a close association between neurons and the endothelium (28). The BBB is a unique microvascular structure in the brain, which helps maintain the homeostatic microenvironment for normal neuronal functions and controls the passage of material between blood and the brain. Cerebral I/R injury states impair endothelial function, which may produce vasogenic brain edema, hemorrhagic transformation and BBB leakage, leading to neuronal apoptosis and neurological damage $(29,30)$.

The present study demonstrated that the MCAO procedure induced a cerebral I/R injury state with neuronal apoptosis, and an increase in brain water and Evans blue content. MCAO also induced an increase in infarct volume and TUNEL positive cells with enhanced caspase- 3 expression. The NO content in brains and the expression of eNOS were found to be significantly decreased in the Pre-IR group compared with the sham group. eNOS is predominantly expressed in the vasculature, and catalyzes the amino acid L-arginine conversion to $\mathrm{NO}$, thus contributing to the maintenance of endovascular homeostasis (31) and the regulation of cerebral blood flow (32). eNOS polymorphisms are accompanied by decreasing $\mathrm{NO}$, which has been reported to be associated with carotid atherosclerosis (33) or cerebral ischemia (34). This is consistent with the findings of the present study. Previous studies suggested that deficiency in endothelial NO and eNOS may cause cerebrovascular pathology and neurological disease, which is a pathogenesis of cerebral ischemia injury.

Notably, the present data also demonstrated that the miR-155 mRNA levels were changed following MCAO procedure. miR-155, the product of the B-cell integration cluster gene, was identified as a critical miRNA during inflammation or cancer $(35,36)$. Previous studies have indicated that there is a relationship between miR-155 and the endothelium. Liu et al (37) have reported miR-155 reduces 
A

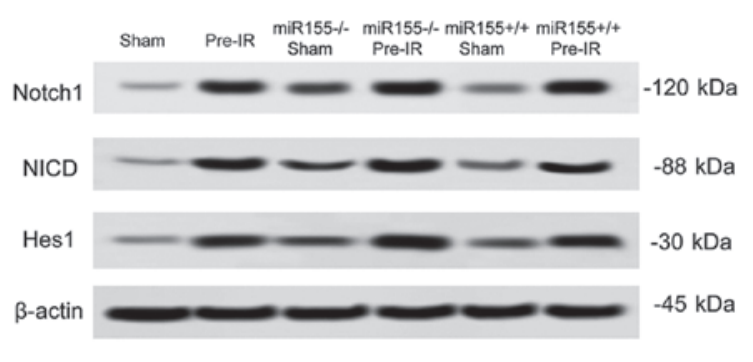

C

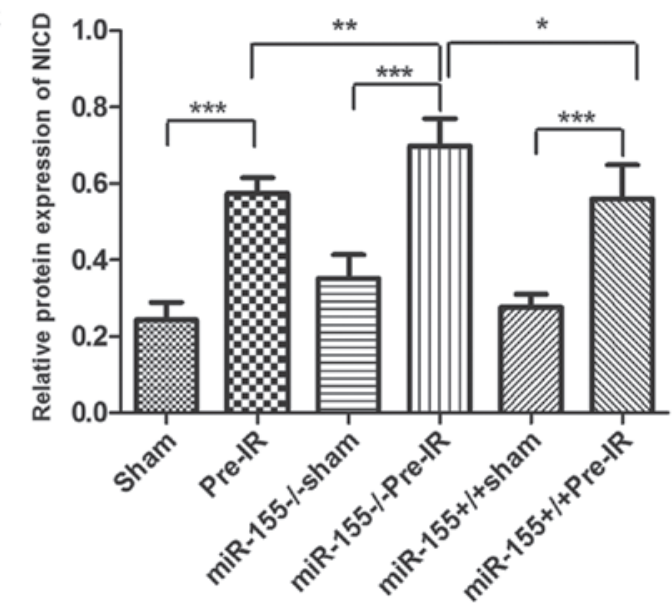

E

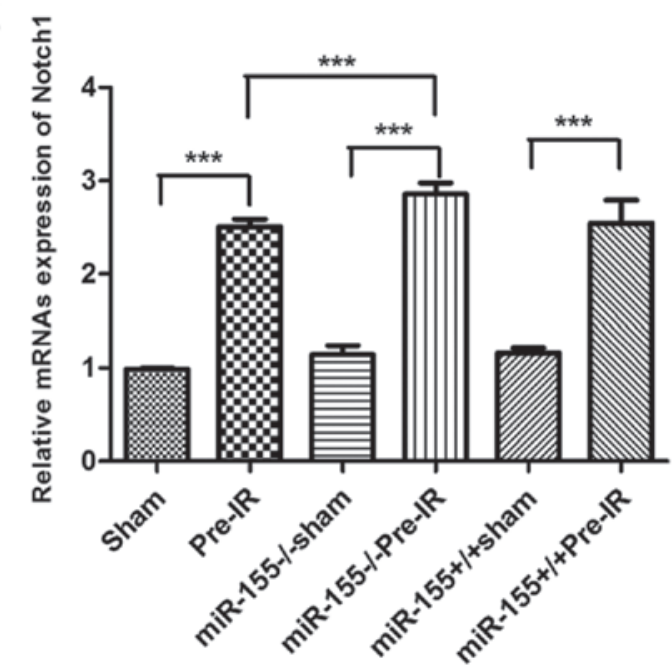

B
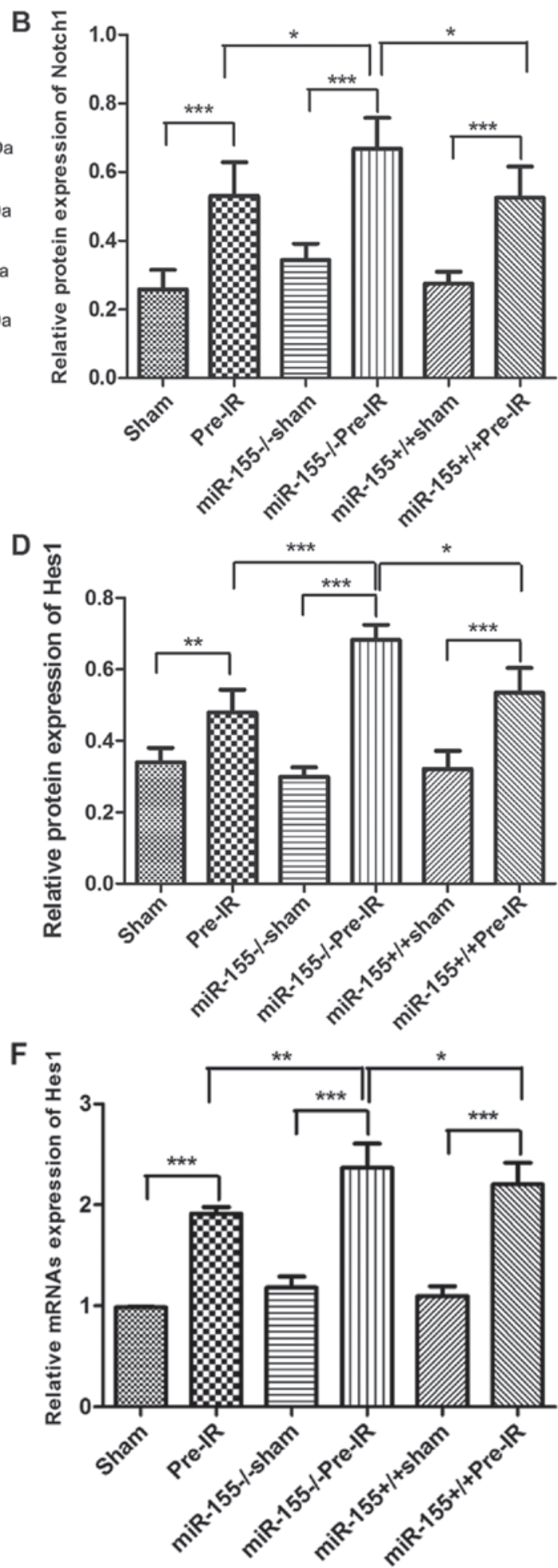

Figure 4. Effects of miR-155 on the Notch signaling pathway following cerebral I/R injury in mice. (A) Protein expression of the Notch pathway in brains at $24 \mathrm{~h}$ following MCAO (n=5). Western blot analysis of Notch1 (B), NICD (C), and Hes1 (D) protein levels in brains. Reverse transcription-quantitative polymerase chain reaction analysis of the (E) Notch1 and (F) Hes1 mRNA levels in brains at $24 \mathrm{~h}$ following MCAO $(\mathrm{n}=5)$. Data are expressed as the mean \pm standard deviation. ${ }^{*} \mathrm{P}<0.05,{ }^{* *} \mathrm{P}<0.01,{ }^{* * * *} \mathrm{P}<0.001$. miR, microRNA; I/R, ischemia reperfusion; MCAO, middle cerebral arterial occlusion; NICD, intracellular Notch

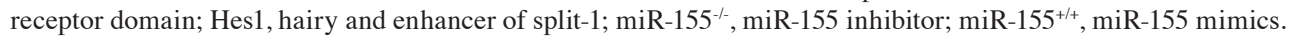

umbilical vein endothelial cells damage via regulating exogenous angiotensin II. However, Huang et al (38) reported that the overexpression of miR-155 contributed to inflammation-mediated glomerular endothelial injury in diabetic nephropathy. In addition, Gama-Norton et al (39) have demonstrated that the Notch signal pathway controls cell fate in the hemogenic endothelium. Ramasamy et al (12) have indicated that Notch signaling promotes endothelial cell proliferation and vessel growth in postnatal long bone. A previous study have also reported there is an association between miR-155 and Notch signaling, Notch/recombination signal binding protein immunoglobulin $\mathrm{\kappa J}$-signaling affects miR-155 in bone 
marrow endothelial cells, leading to $\mathrm{NF}-\kappa \mathrm{B}$ activation and increased proinflammatory cytokine production (40). It was therefore hypothesized that the cerebral I/R induced neuronal apoptosis and BBB damage may be associated with miR-155 and the Notch signaling pathway. Therefore, a major aim of the present study was to determine whether there is an association between miR-155 and the Notch signaling pathway.

The results of the present study indicated that deletion of miR-155 raised the expression of Notch1, NICD and Hes1, thus activating the Notch signaling pathway. In addition, the percentage of TUNEL-positive cells and caspase-3 levels were decreased, with alleviated neuronal apoptosis. These findings indicated that miR-155 may be an essential regulator of the Notch signaling pathway.

Additionally, there were a number of limitations in the present study. It is not fully clear yet if Notch1 is directly targeted by miR-155, due to the absence of in vitro experiments and detailed molecular mechanism research in the present study. However, this limitation also provides a direction for future research, and more work is needed to detect and explore the mechanism underlying the miR155 effects on the Notch signaling pathway.

Furthermore, it was hypothesized that miR-155 downregulates eNOS expression mainly through decreasing the Notch pathway. The Notch pathway is important in controlling vascular smooth muscle cells, and is also critical to vascular development, repair, and remodeling (41-44). NICD may move into the signal-receiving cell nucleus and regulates the expression of downstream target genes, such as Hes1 (45). Yu et al (46) recently reported that the activation of Notch1 and Hes1 is instrumental in withstanding myocardial I/R injury. Tu et al (47) previously demonstrated that the activated Notch signaling pathway has been involved in the arteriovenous lesions in mice in accordance with the present conjecture. The present findings suggested that the activation of the Notch pathway upregulated the eNOS expression and NO production and alleviated cerebral ischemic injury, resulting in cerebrovascular pathology and neurological disease.

The present study demonstrated that miR-155 was involved in the negative regulation of NO production and eNOS expression via the Notch signaling pathway. Deletion of miR-155 contributed to cerebral I/R injury-induced decreases of NO production, eNOS expression and neuronal apoptosis through upregulation of Notch1, NICD and Hes1. Overexpression of miR-155 aggravated BBB leakage and neuronal apoptosis, and downregulation of Notch1, NICD and Hes1. These findings suggest that miR-155 serves an essential role in regulating the Notch signaling pathway of the nervous system. Furthermore, these findings lay a foundation for using miR-155 in the treatment of cerebral stroke.

\section{Acknowledgements}

Not applicable.

\section{Funding}

The present study was supported by a grant from the Guizhou Province Science and Technology Project (grant no. Qiankehe Jichu [2016] 1126).

\section{Availability of data and materials}

The datasets used and/or analyzed during the present study are available from the corresponding author on reasonable request.

\section{Authors' contributions}

TJ constructed the middle cerebral artery occlusion model and performed western blotting. SZ and XL performed the histological analysis and statistical analysis. JS and TA conducted the 2-3-5-triphenyl terazolium chloride staining and measured the brain water content. XH and XP conducted the TUNEL staining and reverse transcription-quantitative polymerase chain reaction analysis. LW was responsible for the design of the study and drafting the manuscript. All authors read and approved the final manuscript.

\section{Ethics approval and consent to participate}

All procedures were approved by the Animal Care and Research Committee of The Affiliated Hospital of Guizhou Medical University (Guiyang, China).

\section{Patient consent for publication}

Not applicable.

\section{Competing interests}

The authors declare that they have no competing interests.

\section{References}

1. Donnan GA, Fisher M, Macleod M and Davis SM: Stroke. Lancet 371: 1612-1623, 2008

2. Cabral NL, Nagel V, Conforto AB, Magalhaes PS, Venancio VG, Safanelli J, Ibiapina F, Mazin S, França P, Liberato RM, et al: High five-year mortality rates of ischemic stroke subtypes: A prospective cohort study in Brazil. Int J Stroke: Oct 9, 2018 (Epub ahead of print).

3. Zhang HT, Zhang P, Gao Y, Li CL, Wang HJ, Chen LC, Feng Y, Li RY, Li YL and Jiang CL: Early VEGF inhibition attenuates blood-brain barrier disruption in ischemic rat brains by regulating the expression of MMPs. Mol Med Rep 15: 57-64, 2017.

4. Kalogeris T, Baines CP, Krenz M and Korthuis RJ: Cell biology of ischemia/reperfusion injury. Int Rev Cell Mol Biol 298: 229-317, 2012.

5. Artavanis-Tsakonas S, Rand MD and Lake RJ: Notch signaling: Cell fate control and signal integration in development. Science 284: 770-776, 1999.

6. Andersson ER, Sandberg R and Lendahl U: Notch signaling: Simplicity in design, versatility in function. Development 138 : 3593-3612, 2011

7. Bray SJ: Notch signalling in context. Nat Rev Mol Cell Biol 17: 722-735, 2016.

8. Yoon K and Gaiano N: Notch signaling in the mammalian central nervous system: Insights from mouse mutants. Nat Neurosci 8: 709-715, 2005.

9. Sun F, Mao X, Xie L, Ding M, Shao B and Jin K: Notch1 signaling modulates neuronal progenitor activity in the subventricular zone in response to aging and focal ischemia. Aging Cell 12: 978-987, 2013.

10. LeComte MD, Shimada IS, Sherwin C and Spees JL: Notch1-STAT3-ETBR signaling axis controls reactive astrocyte proliferation after brain injury. Proc Natl Acad Sci USA 112: 8726-8731, 2015.

11. Pei H, Song X, Peng C, Tan Y, Li Y,Li X, Ma S, Wang Q, Huang R, Yang D, et al: TNF- $\alpha$ inhibitor protects against myocardial ischemia/reperfusion injury via Notch1-mediated suppression of oxidative/nitrative stress. Free Radic Biol Med 82: 114-121, 2015. 
12. Ramasamy SK, Kusumbe AP, Wang L and Adams RH: Endothelial Notch activity promotes angiogenesis and osteogenesis in bone. Nature 507: 376-380, 2014

13. Patenaude A, Fuller M, Chang L, Wong F, Paliouras G, Shaw R, Kyle AH, Umlandt P, Baker JH, Diaz E, et al: Endothelial-specific Notch blockade inhibits vascular function and tumor growth through an eNOS-dependent mechanism. Cancer Res 74: 2402-2411, 2014.

14. Ambros V: The functions of animal microRNAs. Nature 431 350-355, 2004

15. Bartel DP: MicroRNAs: Genomics, biogenesis, mechanism, and function. Cell 116: 281-297, 2004.

16. Sepramaniam S, Tan JR, Tan KS, DeSilva DA, Tavintharan S, Woon FP, Wang CW, Yong FL, Karolina DS, Kaur P, et al: Circulating microRNAs as biomarkers of acute stroke. Int J Mol Sci 15: 1418-1432, 2014

17. Liu DZ, Tian Y, Ander BP, Xu H, Stamova BS, Zhan X, Turner RJ, Jickling $G$ and Sharp FR: Brain and blood microRNA expression profiling of ischemic stroke, intracerebral hemorrhage, and kainate seizures. J Cereb Blood Flow Metab 30: 92-101, 2010.

18. Li SH, Su SY and Liu JL: Differential regulation of microRNAs in patients with ischemic stroke. Curr Neurovasc Res 12: 214-221, 2015.

19. Caballero-Garrido E, Pena-Philippides JC, Lordkipanidze T, Bragin D, Yang Y, Erhardt EB and Roitbak T: In vivo inhibition of miR-155 promotes recovery after experimental mouse stroke. J Neurosci 35: 12446-12464, 2015.

20. Choi GH, Ko KH, Kim JO, Kim J, Oh SH, Ha IB, Cho KG, Kim OJ, Bae J and Kim NK: Association of miR-34a, miR-130a, miR-150 and miR-155 polymorphisms with the risk of ischemic stroke. Int J Mol Med 38: 345-356, 2016.

21. Sun HX, Zeng DY, Li RT, Pang RP, Yang H, Hu YL, Zhang Q, Jiang Y, Huang LY, Tang YB, et al: Essential role of microRNA-155 in regulating endothelium-dependent vasorelaxation by targeting endothelial nitric oxide synthase. Hypertension 60: 1407-1414, 2012.

22. Cerutti C, Soblechero-Martin P, Wu D, Lopez-Ramirez MA, de Vries H, Sharrack B, Male DK and Romero IA: MicroRNA-155 contributes to shear-resistant leukocyte adhesion to human brain endothelium in vitro. Fluids Barriers CNS 13: 8, 2016

23. Wang Y, Huang J, Ma Y, Tang G, Liu Y, Chen X, Zhang Z, Zeng L, Wang Y, Ouyang YB and Yang GY: MicroRNA-29b is a therapeutic target in cerebral ischemia associated with aquaporin 4. J Cereb Blood Flow Metab 35: 1977-1984, 2015.

24. Hara H, Friedlander RM, Gagliardini V, Ayata C, Fink K, Huang Z, Shimizu-Sasamata M, Yuan J and Moskowitz MA: Inhibition of interleukin 1beta converting enzyme family proteases reduces ischemic and excitotoxic neuronal damage. Proc Natl Acad Sci USA 94: 2007-2012, 1997.

25. Li Y, Hua X, Hua F, Mao W, Wan L and Li S: Are bone marrow regenerative cells ideal seed cells for the treatment of cerebral ischemia? Neural Regen Res 8: 1201-1209, 2013.

26. Xia P, Chen HY, Chen SF, Wang L, Strappe PM, Yang HL, Zhou CH, Zhang X, Zhang YX, Ma LL and Wang L: The stimulatory effects of eNOS/F92A-Cav1 on NO production and angiogenesis in BMSCs. Biomed Pharmacother 77: 7-13, 2016.

27. Livak KJ and Schmittgen TD: Analysis of relative gene expression data using real-time quantitative PCR and the 2(-Delta Delta C(T)) method. Methods 25: 402-408, 2001.

28. del Zoppo GJ and Mabuchi T: Cerebral microvessel responses to focal ischemia. J Cereb Blood Flow Metab 23: 879-894, 2003.

29. Obermeier B, Daneman R and Ransohoff RM: Development, maintenance and disruption of the blood-brain barrier. Nat Med 19: 1584-1596, 2013

30. Moskowitz MA, Lo EH and Iadecola C: The science of stroke: Mechanisms in search of treatments. Neuron 67: 181-198, 2010.

31. Srivastava K, Bath PM and Bayraktutan U: Current therapeutic strategies to mitigate the eNOS dysfunction in ischaemic stroke. Cell Mol Neurobiol 32: 319-336, 2012.
32. Milsom AB, Patel NS, Mazzon E, Tripatara P, Storey A, Mota-Filipe H, Sepodes B, Webb AJ, Cuzzocrea S, Hobbs AJ, et al: Role for endothelial nitric oxide synthase in nitrite-induced protection against renal ischemia-reperfusion injury in mice. Nitric Oxide 22: 141-148, 2010.

33. Fatini C, Sofi F, Gensini F, Sticchi E, Lari B, Pratesi G, Pulli R, Dorigo W, Pratesi C, Gensini GF and Abbate R: Influence of eNOS gene polymorphisms on carotid atherosclerosis. Eur J Vasc Endovasc Surg 27: 540-544, 2004.

34. Atochin DN, Clark J, Demchenko IT, Moskowitz MA and Huang PL: Rapid cerebral ischemic preconditioning in mice deficient in endothelial and neuronal nitric oxide synthases. Stroke 34: 1299-1303, 2003

35. Woodbury ME, Freilich RW, Cheng CJ, Asai H, Ikezu S, Boucher JD, Slack F and Ikezu T: miR-155 Is essential for inflammation-induced hippocampal neurogenic dysfunction. J Neurosci 35: 9764-9781, 2015.

36. Chen S, Wang L, Fan J, Ye C, Dominguez D, Zhang Y, Curiel TJ, Fang D, Kuzel TM and Zhang B: Host miR155 promotes tumor growth through a myeloid-derived suppressor cell-dependent mechanism. Cancer Res 75: 519-531, 2015.

37. Liu T, Shen D, Xing S, Chen J, Yu Z, Wang J, Wu B, Chi H, Zhao H, Liang Z and Chen C: Attenuation of exogenous angiotensin II stress-induced damage and apoptosis in human vascular endothelial cells via microRNA-155 expression. Int J Mol Med 31: 188-196, 2013.

38. Huang Y, Liu Y, Li L, Su B, Yang L, Fan W, Yin Q, Chen L, Cui T, Zhang J,et al: Involvement of inflammation-related miR-155 and miR-146a in diabetic nephropathy: Implications for glomerular endothelial injury. BMC Nephrol 15: 142, 2014.

39. Gama-Norton L, Ferrando E, Ruiz-Herguido C, Liu Z, Guiu J, Islam AB, Lee SU, Yan M, Guidos CJ, López-Bigas N, et al: Notch signal strength controls cell fate in the haemogenic endothelium. Nat Commun 6: 8510, 2015.

40. Wang L, Zhang H, Rodriguez S, Cao L, Parish J, Mumaw C, Zollman A, Kamoka MM, Mu J, Chen DZ, et al: Notch-dependent repression of miR-155 in the bone marrow niche regulates hematopoiesis in an NF- $\mathrm{BB}$-dependent manner. Cell Stem Cell 15: $51-65,2014$

41. Kurpinski K, Lam H, Chu J, Wang A, Kim A, Tsay E, Agrawal S, Schaffer DV and Li S: Transforming growth factor-beta and notch signaling mediate stem cell differentiation into smooth muscle cells. Stem Cells 28: 734-742, 2010

42. Hofmann JJ and Iruela-Arispe ML: Notch signaling in blood vessels: Who is talking to whom about what? Circ Res 100: 1556-1568, 2007

43. Holderfield MT and Hughes CC: Crosstalk between vascular endothelial growth factor, notch, and transforming growth factor-beta in vascular morphogenesis. Circ Res 102: 637-652, 2008

44. Zou S, Ren P, Nguyen M, Coselli JS, Shen YH and LeMaire SA: Notch signaling in descending thoracic aortic aneurysm and dissection. PLoS One 7: e52833, 2012.

45. Yan B, Raben N and Plotz P: The human acid alpha-glucosidase gene is a novel target of the Notch-1/Hes-1 signaling pathway. J Biol Chem 277: 29760-29764, 2002.

46. Yu L, Liang H, Lu Z, Zhao G, Zhai M, Yang Y, Yang J, Yi D, Chen W, Wang X, et al: Membrane receptor-dependent Notch1/Hes1 activation by melatonin protects against myocardial ischemia-reperfusion injury: In vivo and in vitro studies. J Pineal Res 59: 420-433, 2015.

47. Tu J, Li Y, Hu Z and Chen Z: Radiosurgery inhibition of the Notch signaling pathway in a rat model of arteriovenous malformations. J Neurosurg 120: 1385-1396, 2014

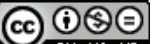

This work is licensed under a Creative Commons Attribution-NonCommercial-NoDerivatives 4.0 International (CC BY-NC-ND 4.0) License. 